\title{
Adenovirus Infection in Children with Diarrhea Disease in Northwestern Nigeria
}

\author{
M. Aminu', A. A. Ahmad ${ }^{1}$, J. U. Umoh', M. C. de Beer ${ }^{3}$, M. D. Esona ${ }^{3}$, A. D. Steele ${ }^{3}$ \\ ${ }^{1}$ Department of Microbiology, Faculty of Science, Ahmadu Bello University, Zaria Nigeria. \\ ${ }^{2}$ Department of Veterinary Public Health and Preventive Medicine, Faculty of Veterinary Medicine, Ahmadu \\ Bello University, Zaria, Nigeria. \\ ${ }^{3}$ MRC/MEDUNSA Diarrhoeal Pathogens Research Unit, University of Limpopo, Medunsa Campus, Pretoria, \\ South Africa. \\ Reprint request to: Dr M. Aminu, Department of Microbiology, Faculty of Science, Ahmadu Bello University, \\ Zaria Nigeria. E-mail: maryamaminu@yahoo.com \\ This study was funded by a grant from UNESCO-L'ORÉAL Fellowship for Women in Life Sciences awarded to Dr. Maryam Aminu. The \\ World Health Organization and the Medical Research Council of South Africa also supported the research.
}

\begin{abstract}
Background: Adenoviruses, particularly enteric adenoviruses (EAds) type 40 (Ad40) and type 41(Ad41), can cause acute and severe diarrhea in young children worldwide. This study was conducted to delineate the epidemiological features of adenoviruses identified in children with gastroenteritis in Northwestern Nigeria.

Methods: All 282 specimens comprising 248 diarrheic and 34 non-diarrheic stools were randomly selected from 1063 stools previously analyzed for rotaviruses. These specimens were collected between July 2002 and July 2004 from children $<5$ years of age. The specimens were screened for the presence of adenoviruses using monoclonal antibody-based Enzyme Immuno Assay (EIA), (Adenovirus RIDASCREEN $^{\circledR}$ r-Biopharm, UK) and the positive specimens were further examined for Ad40 and Ad41 using Premier Adenoclone ${ }^{\circledR}$-Type 40/41 EIA (Meridian Biosciences, USA). Negative staining electron microscopy was performed on selected specimens to confirm the presence of adenovirus particles.

Results: Adenovirus antigen was detected in 63/282 (23\%) of the diarrheic diarrheic and in 6/34 (17.6\%) of the non-diarrheic specimens. Adenoviruses were detected throughout the study period with most patients infected in the age group 25-36 months. The male-to-female ratio was 2.2:1 (43/20). Clinical features included fever (60\%: 38/63), vomiting (56\%: 35/63), mild dehydration (49\%: 31/63), symptoms of upper respiratory tract infection $(13 \%$ : 8/63) and abdominal pain (5\%: 3/63). Analysis of stool specimen in adenovirus infected patients showed watery diarrhea in $87 \%(55 / 63)$, diarrhea with mucus in $19 \%(12 / 63)$ and diarrhea with mucus and blood in 3\% (2/63). Ten (10) percent of the children were hospitalized due to gastroenteritis while 9 patients $(14.3 \%)$ had co-infections with rotavirus. Human EAds were detected in $8 \%$ of specimens mainly in the dry season and among children older than 2 years. The principal symptoms were diarrhea $(100 \%)$, dehydration $(80 \%)$ and fever $(80 \%)$.

Conclusion: The findings of this study suggest that adenoviruses are important etiologic agents of gastroenteritis in Northwestern Nigerian children.
\end{abstract}

Key words: Adenovirus, diarrhea, clinical features, children, Nigeria

\section{Résumé}

Introduction: Adénoviruses, les adénovirus entériques (Eads) de type 40 (Ad40) et le type 41(Ad41), en particulier, peuvent provoquer des graves diarrhées aigues chez les jeunes enfants partout dans le monde. Cette étude a été réalisée pour délimiter les caractéristiques épidémiologiques des adénovirus identifiées chez les enfants avec gastro-entérite dans le nord-ouest du Nigéria.

Méthodes: Tous les 282 échantillons comprenant 248 diarrhéiques et 34 selles non diarrhéiques ont été choisis au hasard à partir de 1063 selles précédemment analysés pour des rotavirues. Ces échantillons ont été recueillis entre juillet 2002 et juillet 2004 chez des enfants de moins de 5 ans. Les échantillons ont été examinés pour la présence d'adénovirus avec l'utilisation d'anticorps monoclonaux à base d'enzyme immunoAssay (EIA,) (Adénovirus Ridascreen@r-Biophram, R.U) et les échantillons positifs ont été encore étudiés pour Ad40 et Ad41 tout en utilisant Adénoclone R Premier - Type 40/41 EIA 
(Biosciences Méridien, USA). Coloration négative microscopie électronique a été opérée sur des échantillons sélectionnées afin de confirmer la présence des particules d'adénovirus.

Résultats: L'antigène d'Adénovirus a été détecté en $63 / 282$ soit $23 \%$ de la diarrhéique et $6 / 34$ soit 17,6\% des échantillons non-diarrhoeques. Adénovirus ont été détectés tout au long de la période d'étude avec la plupart des patients infectés dans le groupe d'âge 25-36 mois. La proportion de l'homme et de la femme était de 2:2 (43/20). Les caractéristiques cliniques inclus: la fièvre (60\%: 38/63), les vomissements $(56 \%$ ; $35 / 63)$ Déshydratation muld (49\%:31/63). Symptômes d'infection des voies respiratoires supérieurs (13\%:8/63) et la douleur d'abdomen (5\%:3/63). L'analyse d'échantillons des selles chez des patients atteints d'adénovirus a montré la diarrhée aqueuse en $87 \%$ (55/63), de la diarrhée avec du mucus, en 19\% $(12 / 63)$ et de la diarrhée avec du mucus et du sang en 3\% (2/63). Dix (10) pourcent des enfants étaient hospitalisés attribuable à la gastro-entérite tandis que 9 malades soit 14,3\% avaient des co-infections avec le rotavirus. EAds humains ont été détectés dans $8 \%$ des échantillons principalement pendant la saison sèche, et parmi des enfants âgés de plus de 2 ans. Des symptômes principaux étaient diarrhée $(100 \%)$, déshydratation $(80 \%)$ et la fièvre $(80 \%)$.

Conclusion: Les résultats de cette étude indiquent que les adénovirus sont des agents étiologiques importants des gastroentérites chez des enfant du nord-ouest du Nigeria.

Mots clés: Adénovirus, des diarrhées, des cliniques, des enfants, le Nigeria

\section{Introduction}

Adenoviruses belonging to the Mastadenovirus genus in the family Adenoviridae cause a variety of diseases and are prevalent throughout the world. Common clinical manifestations resulting from adenovirus infection include pneumonia, cystitis, conjunctivitis, diarrhea, hepatitis, myocarditis, intussusception and encephalitis. $^{1,2}$ Adenoviruses are increasingly recognized as agents of life-threatening infection in immunocompromised patients, particularly in human immunodeficiency virus positive individuals and allogeneic bone marrow transplant recipients often with very high mortality. ${ }^{2,3}$ To date, 51 human adenovirus serotypes that are divided into six species (A to F) based on their ability to agglutinate various types of erythrocytes are distinguished. ${ }^{4}$ Although, adenoviruses are more often associated with respiratory infections, some serotypes, denominated enteric adenoviruses (EAds) are related to diarrheal disease. The adenoviruses most commonly associated with diarrheal illnesses or gastroenteritis in young children includes those of species $\mathrm{F}$ (serotypes 40 and 41) and species A (serotypes 12, 18 and 31). However, Ad40 and Ad41 primarily affect the gut, contributing to $5 \%-20 \%$ of hospitalizations for childhood diarrhea. ${ }^{5}$

The EAds are a relatively unknown entity in gastroenteritis epidemiology in Africa with the exception of South Africa where their etiological importance in pediatric gastroenteritis has been established. $^{6-8}$ However, indications are that the detection of adenovirus in diarrheal stools is probably under-reported due to low or lack of specificity of commercially available EIA for all circulating adenovirus strains. ${ }^{6}$ This is speculated to be true for most of African adenovirus strains. ${ }^{7}$

In Nigeria, few available studies conducted in the Southern and Northcentral regions have associated adenoviruses with $3.8 \%{ }^{9}$ and $6.7 \%{ }^{10}$ of pediatric diarrhea. In Northwestern Nigeria, there is no published information on the epidemiology of EAds which some studies ${ }^{11-13}$ have implicated as the second most important viral agent associated with gastroenteritis in children. This study was therefore conducted to investigate the epidemiology of human enteric adenoviruses associated with gastroenteritis in children 0-5 years old in Northwestern Nigeria.

\section{Materials and Methods}

\section{Study area and population}

This study was conducted in four states (Kaduna, Kebbi, Sokoto and Zamfara) in Northwestern Nigeria between July 2002 and July 2004. The region has a typical tropical continental climate and Northern dry savannah vegetation with alternating humid to wet and cool to hot dry season (April to September and October to March respectively). The region is urban with people who are mainly Muslims with different educational and socio-economic background living in neighborhoods with distinctly different levels of sanitation. Borehole and well water are the major sources of drinking water in these areas. The main occupations here are cattle rearing and subsistence farming. All the state headquarters have Government run hospitals, private clinics and Primary Health Care Units (PHCU) that provide health services to the population. The health care facilities of these state headquarters serve all the surrounding villages in each state. The study was approved by each State Ministry of Health and the ethics committee of each clinic/hospital.

The study population comprised of 282 stool specimens randomly selected from 1063 stool specimen that were previously analyzed for rotaviruses using commercially available EIA (Rotavirus IDEIA ${ }^{\mathrm{TM}}$, DAKO, UK). These stool specimens were collected from children under the age of 5 years who were presented or admitted at clinics or hospitals for acute diarrheal illness in the four states. Specimens from 34 non-diarrheic (control) and 6 intussusception patients were included in the collection. A diarrheic case in this study was defined as a child passing loose, liquid, watery or a bloody 
loose stool three or more times in a 24-hour period as reported by parents. The control was any child presented for an illness other than diarrhea and with no history of it on the day of, or in the three weeks preceding sampling.

\section{Collection and storage of specimens}

At the respective health care facility, arrangements were made with a doctor, matron or laboratory technician whereby the parent/caregiver of any child who satisfies the study inclusion criteria (age 0-5 years of both sexes and diarrheic) was asked to provide the child's stool specimen after consultation. Prior to collection of specimen, the parent/caregiver after consent was interviewed using structured questionnaire designed to obtain basic demographic data, history of illness and clinical information concerning the child. Information on the clinical features and the dehydration status were provided by the Doctor/matron on duty or obtained from the hospital folder of each child. Faecal specimens were collected in clean, labeled screw capped tubes and were stored frozen $\left(-20^{\circ} \mathrm{C}\right)$ at the Department of Microbiology, Ahmadu Bello University, Zaria, Nigeria, until transportation. Only one stool specimen was collected per child. Specimens within iceboxes were transported to the MRC/MEDUNSA Diarrheal Pathogens Research Unit, University of Limpopo, Medunsa Campus, Pretoria, South Africa, where a $10 \%$ fecal suspension of each specimen was prepared using a balanced salt solution before analysis.

\section{Adenovirus antigen enzyme immunoassay (EIA)}

All 282 stool specimens comprising of 248 diarrheic and 34 non-diarrheic specimens were screened for adenoviruses using commercially available genusspecific kits (Adenovirus RIDASCREEN ${ }^{\circledR}$ rBiopharm, UK) according to the manufacturer's instructions. Results were interpreted against control included in each assay. Briefly, a $10 \%$ dilution of each specimen was prepared, mixed thoroughly with a vortex mixer and allowed to settle for about 30 minutes prior to testing. Approximately $100 \mu \mathrm{l}$ of each diluted specimen, a positive (inactivated adenovirus strain) and a negative control (distilled water) were dispensed into each microwell of a 96-well microtiter plate pre-coated with mouse monoclonal antibody (MAb) against group specific antigen of adenovirus. This was followed by adding $100 \mu 1$ of enzyme conjugate (adenovirus specific mouse MAb conjugated to horseradish peroxidase) to each microwell and incubation for 1 hour at room temperature. After a total of five washes with distilled water, $100 \mu 1$ of both substrate Part A (tetramethylbenzidine, TMB in a substrate buffer) and $B$ (hydrogen peroxide) was added into each microwell followed by incubation at room temperature for 15 minutes in the dark. Reactivity was determined by spectrophotometry at $450 \mathrm{~nm}$ after adding stopping solution (1 $\mathrm{N}$ sulfuric acid). An absorbance equal to or greater than 0.150 units was considered positive.

\section{Adenoclone (40 and 41) EIA}

The positive adenovirus specimens from the RIDASCREEN $^{\circledR}$ assay were further examined for human EAds serotypes 40 and 41 using a second species-specific EIA kit, Premier Adenoclone ${ }^{\circledR}$-Type 40/41 (Meridian Biosciences, USA). This assay was performed according to the manufacturer's specification using a 48-well microtiter plate and enzyme conjugated to anti-adenovirus types 40 and 41 murine MAbs.

\section{Electron microscopy}

Negative staining electron microscopy (EM) was performed on the positive EAds specimens to further confirm the presence of the viral particles. Briefly, the fresh stool specimens were diluted with distilled water, stained with 3\% phosphotungstic acid (PTA) and placed on grids. The grids were examined in a Phillips 301 electron microscope at a magnification of x 45, 000 .

\section{Statistical analysis}

Associations between occurrences of diarrhea, history of hospitalization, state from where the specimens were obtained, socio-economic status, information with rotavirus and information with adenovirus were analyzed using either odds ratio (OR) with 95\% confidence intervals (CI) or $\chi^{2}$ analysis using SPSS 11.0 program.

\section{Results}

\section{Adenovirus antigen}

Adenoviruses were detected in $22.3 \%(63 / 282)$ of the total specimens. Adenovirus antigen was detected in $23 \%(57 / 248)$ of the diarrheic and $17.6 \%(6 / 34)$ of the non-diarrheic specimens. Adenovirus infection occurred with a higher incidence in the outpatients (24.2\%: 57/236) than inpatients (13.0\%: 6/46). Furthermore, the outpatients were two times more likely to have been infected with adenovirus than the inpatients $(\mathrm{OR}=2.123,95 \% \mathrm{CI}=0.856-5.265)$.

Analysis of adenovirus infection by state showed the highest incidence in Sokoto State (32.5\%: 13/40), followed by Kebbi State (25.0\%: 4/16) (Table 1). Viral shedding occurred significantly more in males $(26.7 \%$ : 43/161) than in females $(16.5 \%$ : $20 / 121)(P<.05)$. Adenovirus infection occurred with the highest incidence in children in the age group 2536 months old (31.0\%: 9/29) followed by those in age group 37-48 months old (30\%: 3/10) (Table 2). The virus occurred more frequently in children above 25 months old with an incidence of $29.2 \%$ (14/48) compared to $21 \%(49 / 234)$ in children under 24 months. Adenoviruses were detected throughout the year except in the month of June, demonstrating no major seasonal variation in the incidence despite some seasonal cluster during the rainy season (April to October) (Figure 1).

A total of 57 out of 63 episodes of infections 
(90.5\%) were accompanied by diarrhea (Table $3)$.Faecal specimens were watery in $87.3 \%(55 / 63)$ of patients, contained mucus in $19.0 \%(12 / 63)$ of patients and mucus and blood in $3.2 \%(2 / 63)$. About $60 \%$ and $56 \%$ of patients experienced fever and vomiting respectively, while $43 \%$ experienced both. Mild dehydration and symptoms of upper respiratory tract infection were also common symptoms in $49 \%$ and $13 \%$ of patients (Table 3). Detection of adenovirus in the stool specimen was possible from the first day to the seventh day of diarrhea and even in diarrhea that has persisted for 3 months. About 47.6\% $(30 / 63)$ of patients had diarrhea duration lasting 1-3 days, $17.5 \%(11 / 63)$ had diarrhea duration lasting for 7 days while only a case $(1 / 63: 1.6 \%)$ had diarrhea that had lasted for 3 months. There was no significant relationship between diarrhea duration and the presence of adenovirus antigen in stool $(P>.05)$.

Figure 1. Percentage distribution of adenovirus infection by month in Northwestern Nigeria

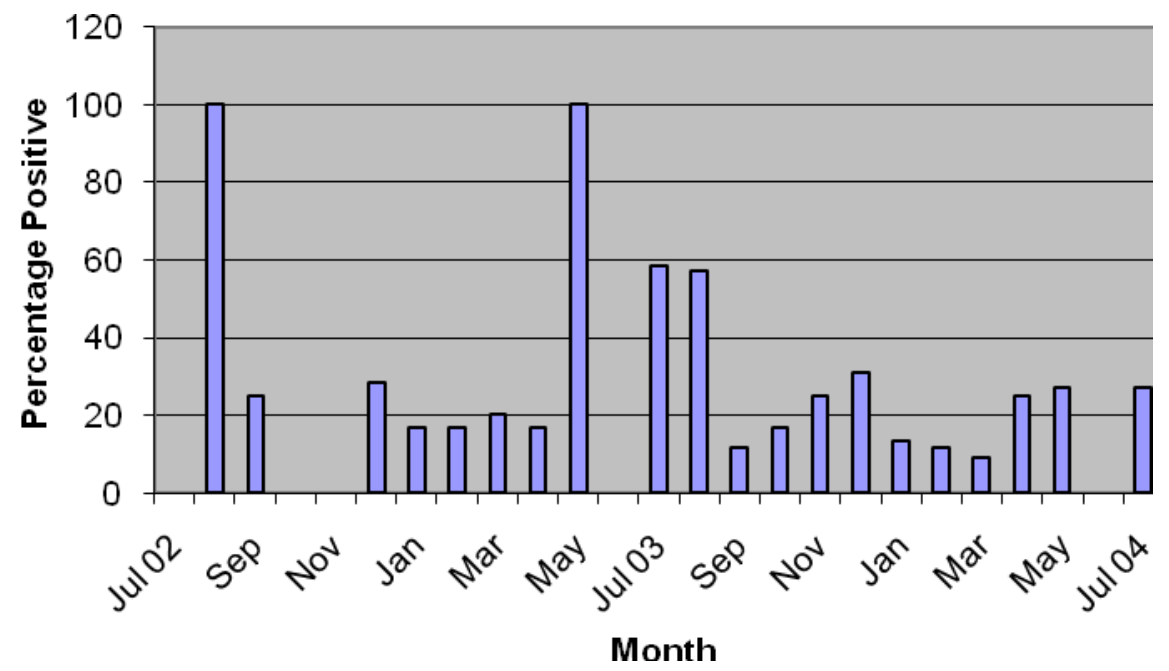

Table 1. Percentage distribution of adenovirus infection by state in Northwestern Nigeria

\begin{tabular}{lll}
\hline \multirow{2}{*}{ State } & \multicolumn{2}{l}{ Adenovirus Antigen } \\
\cline { 2 - 3 } & Total & No. (\%) \\
\cline { 2 - 3 } Kaduna & 156 & $32(20.5)$ \\
Kebbi & 16 & $4(25.0)$ \\
Sokoto & 40 & $\mathbf{1 3}(\mathbf{3 2 . 5})$ \\
Zamfara & 70 & $14(20.0)$ \\
\hline Total & $\mathbf{2 8 2}$ & $\mathbf{6 3 ( 2 2 . 3 )}$ \\
\hline
\end{tabular}

Table 2. Age distribution of children with adenovirus infection in Northwestern Nigeria

\begin{tabular}{lll}
\hline $\begin{array}{l}\text { Age group } \\
\text { (months) }\end{array}$ & No. tested & $\begin{array}{l}\text { No. of cases } \\
(\%)\end{array}$ \\
\hline $0-6$ & 74 & $14(19.0)$ \\
$7-12$ & 91 & $22(24.2)$ \\
$13-18$ & 31 & $7(22.6)$ \\
$19-24$ & 38 & $6(15.8)$ \\
$25-36$ & 29 & $9(31.0)$ \\
$37-48$ & 10 & $3(30.0)$ \\
$49-60$ & 9 & $2(22.2)$ \\
\hline Total & $\mathbf{2 8 2}$ & $\mathbf{6 3 ( 2 2 . 3 )}$ \\
\hline
\end{tabular}

Analysis of the outcome of visit showed that $25 \%$ of adenovirus infected children required treatment, $10 \%$ required hospitalization on visit, and $25 \%$ were sent to the laboratory for stool analysis and then subsequently treated while the only referred child was not shedding adenovirus. There was a significant association between outcome of visit and adenovirus infection $(P<.05)$. The treated children were three times more likely to have been infected with adenovirus than those hospitalized $(\mathrm{OR}=3.000,95 \%$ $\mathrm{CI}=1.137-7.918)$. There were no significant relationships between socioeconomic background, type of drinking water and feeding regimen of a child and adenovirus infection $(P>.05)$.

Table 3. Clinical manifestation of enteric adenovirus infection in children in Northwestern Nigeria $(n=63)$

\begin{tabular}{ll}
\hline Symptom/sign & No. (\%) \\
\hline Diarrhea & $57(90.5)$ \\
Watery stool & $55(87.3)$ \\
Fever & $38(60.0)$ \\
Vomiting & $35(55.6)$ \\
Dehydration (mild) & $31(49.2)$ \\
Fever and vomiting & $27(42.9)$ \\
Stool with mucus & $12(19.0)$ \\
Symptoms of URTI & $8(12.7)$ \\
Abdominal pain & $3(4.8)$ \\
Stool with mucus and blood & $2(3.2)$ \\
Eye infection & $1(1.6)$ \\
\hline
\end{tabular}

URTI: Upper respiratory tract infection 
Co-infection with rotaviruses was observed in $14.3 \%(9 / 63)$ of adenovirus infected patients. Almost all (89\%: 8/9) of the co-infected children were from the diarrheic population. There were no significant relationships between co-infection and the prevalence of clinical symptoms observed in these children despite the differences in prevalence. Children with fever were three and half times more likely to have been infected with both viruses than those without fever $(\mathrm{OR}=3.679,95 \% \mathrm{CI}=0.453$ 29.879), while those who experienced vomiting were five and half times more likely to have been infected $(\mathrm{OR}=5.565,95 \% \mathrm{CI}=0.686-45.120)$. Two third, $(66.7 \%)$ of these children passed stool continuously and were three and half times more likely to have been infected with both viruses than those who did $\operatorname{not}(\mathrm{OR}=3.515,95 \% \mathrm{CI}=0.860-14.365)$.

\section{Enteric adenoviruses types 40 and 41}

Human EAds types 40 and 41 were detected in $8 \%$ $(5 / 63)$ of the positive specimens. Majority $(80 \%)$ of the EAds were detected among outpatients and more frequently in males than females. The EAds were more frequent among children 24 to 32 months of age $(80 \%)$ with a single case in a 2 -month old child and were detected mainly in the dry season (between October and April). All the 5 episodes of infections were accompanied by diarrhea. Faecal specimens were watery in $80 \%(4 / 5)$ of patients and contained mucus in $20 \%(1 / 5)$. About $80 \%$ and $60 \%$ of patients experienced fever and vomiting respectively while $60 \%$ experienced both. Mild to severe dehydration occurred in $80 \%$ of patients and none of the patient had symptoms of URT infection. Patients had diarrhea duration lasting 2-4 days. Majority (80\%) of EAds infected children required treatment.

\section{Electron microscopy}

Adenovirus was detected in only one of the 10 specimens examined by EM.

\section{Discussion}

The prevalence rate $(22.3 \%)$ of adenovirus infection obtained in this study is much higher than rates previously reported in Nigeria. ${ }^{10,14}$ In Africa, lower prevalence of $3.0 \%, 9.8 \%$ and $7.8 \%$ have been observed. $^{6,13,15}$ A similar prevalence $(23.0 \%)$ was obtained in a study in Tanzania. ${ }^{12}$ Lower prevalence such as, $4.0 \%$ have been reported in Finland, ${ }^{16} 4.9 \%$ in Brazil ${ }^{17}$ and $3.6 \%$ in Denmark. ${ }^{18}$

Adenoviruses were detected in $23.0 \%$ of the diarrheaic and in $17.6 \%$ of the non-diarrheaic specimens. The similar prevalence among the two groups, a finding previously reported in Southern Nigeria ${ }^{14}$ probably reflects the fact that adenoviruses other than EAds are often excreted in the stool. More so, adenoviruses cause a number of other symptoms including respiratory, conjunctivitis and pneumonia that would all be common in infants.

Adenovirus infection was more common in outpatients than inpatients. This has been previously noted. ${ }^{17,19}$ Majority of illness due to adenovirus were mild and were not likely to be associated with severe dehydrating diarrhea that leads to or require hospitalization.

The finding that significantly more of the adenovirus infected children were males than females contrasts a previous report in Nigeria. ${ }^{14}$ It is possible that more boys than girls are infected at infancy, but we consider this unlikely as the chance of exposure to the virus should be similar for both sexes.

The identification of adenovirus mostly in children older than 2 years, contrasts the findings of most studies conducted worldwide where children $<2$ years of age are more vulnerable to adenovirus infection. ${ }^{9,10,13,20}$ This incompatible result may be attributed to breastfeeding, socio economic status, hygiene, culture and climate. Steele et al, ${ }^{15}$ however, in their study found adenovirus to be more prevalent in children 3-30 months old.

Adenoviruses were detected in all the states studied, a finding previously reported, ${ }^{17}$ indicating their wide distribution throughout the zone. It is not surprising that adenovirus were detected throughout the study period since they are associated with different clinical conditions. Co-infection with rotaviruses was observed in $14.3 \%$ of adenovirusinfected patients a finding previously reported but in low frequency. ${ }^{9,21}$ The dual infection observed in this study raise the question of whether a single virus is responsible for illness or whether two viruses act in synergy.

Similar to findings in other African countries, EAds 40 and 41 were detected in $8.0 \%$ of the positive specimens. ${ }^{8,12}$ Uhnoo et $\mathrm{al}^{5}$ and Harsi et $\mathrm{al}^{21}$ reported prevalence rates of $7.9 \%$ and $4.5 \%$ in Sweden and Brazil respectively. The result, however, contrasts previous findings in Nigeria where prevalence of $3.8 \%{ }^{9}$ and $3.6 \%{ }^{14}$ were reported. On the other hand, much lower rates such as $2.0 \%$ in Botswana, ${ }^{13} 1.55 \%$ in Brazil, ${ }^{17} 2.3 \%$ in South Africa ${ }^{21}$ and $0.8 \%$ in the United States ${ }^{22}$ have been observed. Possibly, such divergence occurs as a consequence of using MAbs for EAds characterization or as a result of the study design. It has been demonstrated that these highly specific reagents can fail to detect genomic variation of EAds, probably because of alteration of external neutralization epitopes under immunological pressure. ${ }^{17}$ The EAd is a relatively unknown entity in gastroenteritis epidemiology in Africa with the exception of South Africa where the etiological importance in pediatric gastroenteritis has been established. ${ }^{6-8}$ The prevalence of adenovirus observed in this study; only confirm the speculations of Moore and coworkers, ${ }^{7}$ that the detection of adenovirus strain in stools of African children is probably under-reported due to low or lack of specificity of commercially available EIA for all circulating adenovirus strains.

Detecting $80 \%$ of EAds antigen in children older than 2 years, contrasts report of a previous study conducted where $76.6 \%$ of EAds were detected in children less than 2 years. ${ }^{23}$ The detection of adenoviruses in only $10 \%$ of specimens by EM maybe 
attributable to insufficient amount of viral particle in stool as it has been shown that EM detects $\geq 10^{6}$ particles per $\mathrm{ml}$ and that specimens containing adenoviruses below the detection threshold for EM would be recorded as negative. ${ }^{24}$

\section{Conclusion}

In this first study to delineate the clinical features of EAds gastroenteritis in Northwestern Nigeria, adenoviruses were shown to circulate at a higher prevalence in association with gastroenteritis in children less than five years old. However, the impact of these viruses in Nigeria is yet to be fully determined.

\section{Acknowledgments}

We appreciate the cooperation of all the doctors, nurses, laboratory technician, and parents/caregivers who helpfully provided clinical specimens

\section{References}

1. Baum SG. Adenovirus. In: Mandel GL, Bennett JE, Dolin R (Eds). Principles and Practices of Infectious Diseases. Philadelphia: Churchill Livingstone, 2000;1626-7.

2. Seranti H, Johnson G, Brown M, Petric M, Tellier R. Comprehensive detection and serotyping of human adenoviruses by PCR and sequencing. J Clin Microbiol. 2004;42:39633969.

3. Slatter MA, Read S, Taylor CE, et al. Adenovirus type $\mathrm{F}$ subtype 41 causing disseminated disease following bone marrow transplantation for immunodeficiency. J Clin Microbiol. 2005;43:1462-1464.

4. Rosen I. A hemagglutination inhibitor technique for typing adenoviruses. Am J Hyg. 1960;71:120128.

5. Uhnoo I, Wadell G, Svensson L, Johansson ME. Importance of enteric Adenoviruses 40 and 41 in acute gastroenteritis in infants and young children. J Clin Microbiol. 1984;20:365-372.

6. Moore P, Steele AD, Lecatsas G, Alexander JJ. Characterization of gastroenteritis-associated adenoviruses in South Africa. S Afr Med J. 1998;88:1587-1592.

7. Moore PL, Steele AD, Alexander JJ. Relevance of commercial diagnostic tests to detection of enteric adenovirus infections in South Africa. J Clin Microbiol. 2000;38:1661-1663.

8. Steele AD, Bos P, Lecatsas G. Adenovirus associated gastroenteritis at Ga-Rankuwa Hospital, South Africa. S Afr J Epidemiol Infect. 1990; 5:69-71.

9. Nimzing L, Geyer A, Sebata $T$ et al. Epidemiology of adenoviruses and rotaviruses identified in young children in Jos, Nigeria. S Afr J Epidemiol Infect. 2000; 15: 40-42.
10. Audu R, Omilabu AS, Peenze I, Steele D. Viral diarrhea in young children in Nigeria. Cent Afr $\mathbf{J}$ Med. 2002;48:59-63.

11. Bates PR, Bailey AS, Wood DJ, Morris DJ, Couriel JM. Comparative epidemiology of rotavirus, subgenus $F$ (types 40 and 41) adenovirus and astrovirus gastroenteritis in children. J Med Virol. 1993;39:224-228.

12. Mhalu FS, Myrmel H, Msengi A, Haukenes G. Prevalence of infection with rotavirus and enteric adenoviruses among children in Tanzania. NIPH Ann. 1988;11:3-7.

13. Basu G, Rossouw J, Sebunya TK, et al. Prevalence of rotavirus, adenovirus and astrovirus infection in young children with gastroenteritis in Gaborone, Botswana. East Afr Med J. 2003;80:652-655.

14. Audu R, Omilabu AS, Peenze I, Steele AD. Isolation and identification of adenovirus recovered from stool of children with diarrhea in Lagos, Nigeria. African Journal of Health Sciences. 2002;9:105-111.

15. Steele AD, Basetse HR, Blacklow NR, Herrmann JE. Astrovirus infection in South Africa: a pilot study. Ann Trop Paediatr 1998; 18: 315-319.

16. Ruuska T, Vesikari T. A prospective study of acute diarrhoea in Finnish children from birth to $2 \frac{1}{2}$ years of age. Acta Paediatr Scand. 1991;80:500-507.

17. Soares CC, Volotao EM, Albuquerque MCM, et al. Prevalence of enteric adenoviruses among children with diarrhea in four Brazilian cities. J Clin Virol. 2002;23:171-177.

18. Olesen B, Neimann J, Bottiger B, et al. Etiology of diarrhoea in young children in Denmark: a case-control study. J Clin Microbiol. 2005;43:3636-3641.

19. Mclver CJ, Palombo EA, Doultree JC, et al. Detection of astrovirus gastroenteritis in children. J Virol Methods. 2000;84:99-105.

20. Hársi CM, Rolim DP, Gomes SA, et al. Adenovirus genome types isolated from stools of children with gastroenteritis in São Paulo, Brazil. J Clin Microbiol. 1995;45:127-134.

21. Marx FE, Taylor MB, Grabow WOK. The prevalence of human astrovirus and enteric adenovirus infection in South African patients with gastroenteritis. S Afr J Epidemiol Infect. $1998 ; 13: 5-9$

22. Rodriguez-Baez N. O’brien R, Qiu SQ, Bass DM. Astrovirus, adenovirus, and rotavirus in hospitalised children: prevalence and association with gastroenteritis. J Pediatr Gastroenterol Nutr. 2002;35:64-68. Lin HC, Kao CL, Lu CY, et al. Enteric adenovirus infection in children in Taipei. J Microbiol Immunol Infect. 2000;33:176-180.

23. Grimwood K, Carzino R, Barnes GL, Bishop RF. Patients with enteric adenovirus gastroenteritis admitted to an Australian paediatric teaching hospital from 1981-1992. J Clin Microbiol. 1995;33:131-136. 